\title{
Resistance of phenolic-treated oil palm stem plywood against subterranean termites and white rot decay.
}

\begin{abstract}
The objectives of the study were to evaluate the effectiveness of phenolic resin in protecting oil palm stem (OPS) plywood against both subterranean termites (Coptotermes curvignathus) and white rot fungi (Pycnoporous sanguineus). Specially cooked, Low molecular weight phenol formaldehyde (LMW PF) resin was used to treat the OPS veneer whilst commercial urea formaldehyde (UF) resin was used to bond the phenolic-treated veneer. OPS plywood were produced using two types of lay-up (100\% outer veneer type and $100 \%$ inner veneer type) with adhesive spread rate of $200 \mathrm{~g} / \mathrm{m} 2$. The results show that treatment of OPS veneer with LMW PF has significantly enhanced the resistance of OPS plywood against both termites and white rot fungi. In the termites resistance test, the percentage of weight loss for untreated samples were $19.2 \%$ (outer veneer) and $23.9 \%$ (inner veneer), while for phenolic treated samples were only $10.7 \%$ and $15.8 \%$, respectively. The phenolic treatment was able to enhance the resistance towards termites by $38 \%$ and towards white rot fungi by $62 \%$. The study has shown LMW PF resin can be used to protect OPS plywood from termites and white rot fungi.
\end{abstract}

Keyword: Oil palm stem; Phenolic resin; Plywood; Termites; White rot fungi; Treatment. 